University of Nebraska - Lincoln

DigitalCommons@University of Nebraska - Lincoln

May 2003

\title{
Recording characteristics of perpendicular magnetic media with CoZrNb, NiFeNb, and exchange-pinned NiFeNb soft magnetic underlayers
}

\author{
Min Zheng \\ MMC Technology, Inc., San Jose, California \\ Albert Chekanov \\ MMC Technology, Inc., San Jose, California \\ Geon Choe \\ MMC Technology, Inc., San Jose, California \\ Kenneth E. Johnson \\ MMC Technology, Inc., San Jose, California \\ Lan Gao \\ University of Nebraska - Lincoln \\ See next page for additional authors
}

Follow this and additional works at: https://digitalcommons.unl.edu/physicsliou

Part of the Physics Commons

Zheng, Min; Chekanov, Albert; Choe, Geon; Johnson, Kenneth E.; Gao, Lan; and Liou, Sy_Hwang, "Recording characteristics of perpendicular magnetic media with CoZrNb, NiFeNb, and exchange-pinned NiFeNb soft magnetic underlayers" (2003). Si-Hwang Liou Publications. 77.

https://digitalcommons.unl.edu/physicsliou/77

This Article is brought to you for free and open access by the Research Papers in Physics and Astronomy at DigitalCommons@University of Nebraska - Lincoln. It has been accepted for inclusion in Si-Hwang Liou Publications by an authorized administrator of DigitalCommons@University of Nebraska - Lincoln. 
Authors

Min Zheng, Albert Chekanov, Geon Choe, Kenneth E. Johnson, Lan Gao, and Sy_Hwang Liou 


\title{
Recording characteristics of perpendicular magnetic media with CoZrNb, $\mathrm{NiFeNb}$, and exchange-pinned NiFeNb soft magnetic underlayers
}

\author{
Min Zheng, ${ }^{\text {a) }}$ Albert Chekanov, Geon Choe, and Kenneth E. Johnson \\ MMC Technology, Inc., 2001 Fortune Drive, San Jose, California 95131 \\ Lan Gao and Sy-Hwang Liou \\ Department of Physics and Astronomy, University of Nebraska, Lincoln, Nebraska 68588
}

(Presented on 12 November 2002)

\begin{abstract}
The recording performance of perpendicular media with amorphous CoZrNb, nanocrystalline $\mathrm{NiFeNb}$, and exchange-pinned FeMn/NiFeNb soft magnetic underlayers (SULs) has been studied. A method has been proposed to quantify the spike noises from the SUL. We found that media with higher permeability NiFeNb SULs showed $4 \mathrm{db}$ higher signal-to-noise ratio than media with lower permeability CoZrNb SULs. The exchange-pinned NiFeNb SUL suppresses spike noise and offers good recording performance. Furthermore, the recording frequency dependence of dynamic coercivity $\left(H_{c}\right)$ was strongly affected by the use of different SULs. The increase in dynamic $H_{c}$ with linear density is negligibly small for media with NiFeNb SULs, whereas dynamic $H_{c}$ sharply increases with increasing density for media with CoZrNb SULs. (c) 2003 American Institute of Physics. [DOI: 10.1063/1.1557719]
\end{abstract}

\section{INTRODUCTION}

The soft magnetic underlayer plays a vital role in both writability and data stability of perpendicular magnetic recording (PMR) media. However, domain walls in the SUL also contribute to read back noise. Previous work has been primarily focused on eliminating domains in the SUL to obtain low spike noise PMR media. ${ }^{1-4}$ Our study shows that domain wall control is important, but one has to consider other characteristics such as writability of the SUL for perpendicular recording media design. In addition, this is a comparative study of the recording performance of a complete PMR media structure with and without exchange-pinned SUL. Here, we report a detailed study on the recording characteristics of the media with different types of SUL, $\mathrm{CoZrNb}$, NiFeNb, and exchange-pinned $\mathrm{FeMn} / \mathrm{NiFeNb}$. Among these SULs, CoZrNb has a higher $B_{s}$ of $\sim 1.2 \mathrm{~T}$ and a lower permeability of 600 compared to a lower $B_{s}$ of $\sim 0.8$ $\mathrm{T}$ and a higher permeability of 1600 for $\mathrm{NiFeNb}$. In the case of $\mathrm{FeMn} / \mathrm{NiFeNb}$, hysteresis loops show that $\mathrm{NiFeNb}$ was exchange pinned along the radial direction with $H_{\mathrm{ex}}$ $=15 \mathrm{Oe}$, and the calculated permeability $\left[\mu=B_{s} /\left(H_{e x}\right.\right.$ $\left.\left.+H_{k}\right)\right]$ is $\sim 400$.

\section{EXPERIMENT}

All the media were sputtered onto $\mathrm{Al}-\mathrm{NiP}$ substrates using $\mathrm{dc}$ magnetron sputtering. The top recording layer was kept the same for all media. All SULs were sputtered at room temperature at a thickness of $250 \mathrm{~nm}$. CoZrNb and $\mathrm{NiFeNb}$ SULs were laminated by a 3-nm-thick $\mathrm{C}$ spacer with a total stacking of five layers. When sputtering exchange-pinned $[$ FeMn $(10 \mathrm{~nm}) / \mathrm{NiFeNb}(80 \mathrm{~nm})] 3$ SUL, a 5-nm-thick $\mathrm{NiFeNb}$ layer was sputtered prior to sputtering FeMn to pro-

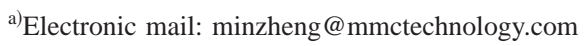

mote a fcc (111) crystalline texture and exchange biasing, which is associated with film growth. The magnetic properties were measured by a vibrating sample magnetometer (VSM). The recording performance was evaluated on a Guzik tester using a single-pole writer and a giant magnetoresistance (GMR) reader.

\section{RESULTS AND DISCUSSIONS}

Up to now, SUL spike noise evaluation was based on observation of the read-signal wave form. This method is simple, but it gives, at best, a qualitative representation. In this work we propose a more direct and quantitative method by counting the number of pulses whose amplitude exceeds a certain threshold. Media performance comparison is done by ranking the pulse count at a fixed threshold level or by rating the slope of the number of pulses versus voltage threshold curve. Figure 1(a) shows a picture demonstrating the method used for counting the number of pulses with amplitude exceeding a certain predefined threshold. Figure 1(b) shows the results at different thresholds on the media with $\mathrm{CoZrNb}$, $\mathrm{NiFeNb}$, and exchange-pinned NiFeNb SULs. The best performing media exhibit a steep noise roll-off with few pulses exceeding the background media noise level, while the high spike noise media show long "tails" extending into the voltage levels exceeding the background noise by a factor of $2-3$. This is a simple and transparent way of performance assessment, and the media performance comparison becomes straightforward. Steeper noise roll-off is observed for the media with exchange-pinned $\mathrm{NiFeNb}$ SUL, indicating that the spike noise is effectively suppressed.

Table I gives a brief summary of the parametric data for these SULs. The measured recording parameters were isolated pulse signals divided by rms media noise $(\mathrm{So} / \mathrm{Nm}), 2 \mathrm{~T}$ signal divided by $2 \mathrm{~T}$ total noise $(\mathrm{SpSNR}), \mathrm{OW}_{1 \mathrm{~T} / 6 \mathrm{~T}}$, and $\mathrm{OW}_{6 \mathrm{~T} / 1 \mathrm{~T}}$. Differentiation of the readback signal was used 


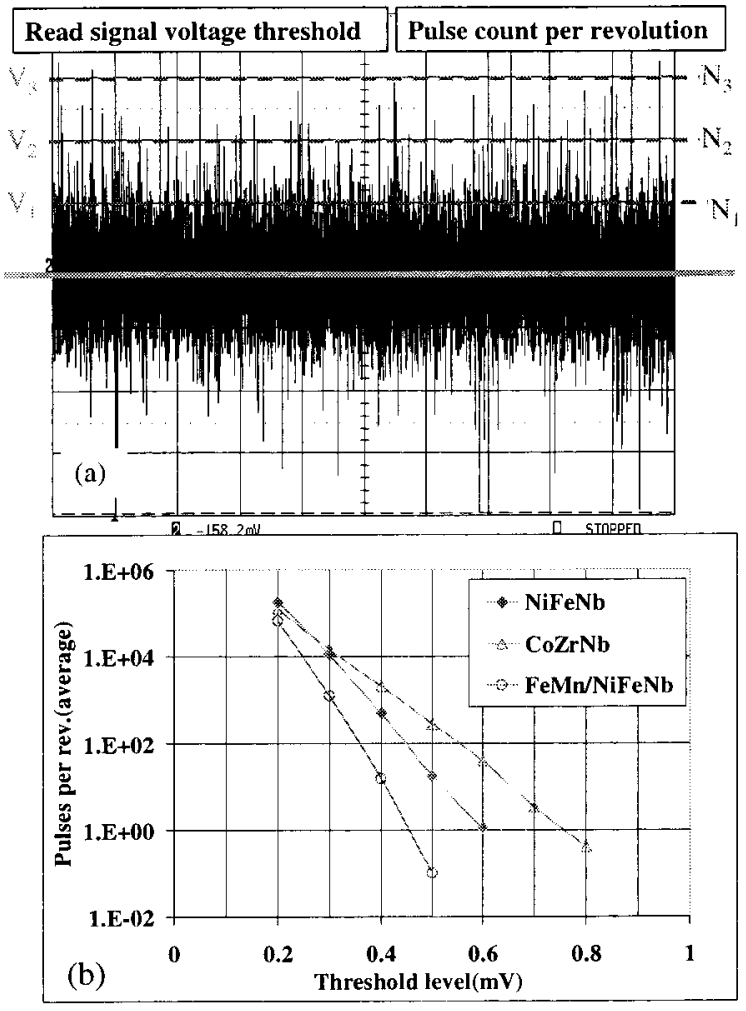

FIG. 1. (a) Method to quantify the spike noise of the SUL. The medium is dc erased and the actual number of pulses is counted with amplitude exceeding a certain predefined threshold, e.g., V1 $(0.2 \mathrm{mV}), \mathrm{V} 2(0.3 \mathrm{mV}), \ldots$, (b) pulses count/revolution vs threshold level for the media with $\mathrm{CoZrNb}$, $\mathrm{NiFeNb}$, and exchange-pinned $\mathrm{NiFeNb}$ SULs.

for measuring $\mathrm{PW}_{50}$. As one can see from Table I, media with NiFeNb SULs show more than $4 \mathrm{~dB}$ higher SpSNR and narrower $\mathrm{PW}_{50}$ than media with CoZrNb SULs. This result is believed to be related to the better field gradient resulting from the superior writability of the NiFeNb SUL media having a higher permeability of 1600 as compared to $\mathrm{CoZrNb}$ with a lower permeability of 600 . The standard overwrite test procedure where the long-wavelength pattern $(6 \mathrm{~T})$ is being overwritten by the short-wavelength pattern $(1 \mathrm{~T})$, i.e., $1 \mathrm{~T} / 6$ $\mathrm{T}$, may be no longer a proper way of characterizing the overwrite in the PMR media due to demagnetization effects. It is more appropriate to use the reversed sequence where overwrite values better reflect the actual head overwrite performance. For example, there is no difference in $\mathrm{OW}_{1 \mathrm{~T} / 6 \mathrm{~T}}$ for

TABLE I. Parametric data for media with different SULs tested at $550 \mathrm{kfci}$. All SULs were kept $250 \mathrm{~nm}$ except the third one, NiFeNb $250 \mathrm{~nm} / \mathrm{CoZrNb}$ $50 \mathrm{~nm}$.

\begin{tabular}{lcccccc}
\hline \hline SUL & $\begin{array}{c}\text { So/Nm } \\
(\mathrm{dB})\end{array}$ & $\begin{array}{c}\text { SpSNR } \\
(\mathrm{dB})\end{array}$ & $\begin{array}{c}\mathrm{OW}_{1 \mathrm{~T} / 6 \mathrm{~T}} \\
(\mathrm{~dB})\end{array}$ & $\begin{array}{c}\mathrm{OW}_{6 \mathrm{~T} / 1 \mathrm{~T}} \\
(\mathrm{~dB})\end{array}$ & $\begin{array}{c}\mathrm{PW}_{50} \\
(\mu \mathrm{in} .)\end{array}$ & $\begin{array}{c}\mathrm{LF} \\
(\mathrm{mV})\end{array}$ \\
\hline $\mathrm{NiFeNb}$ & 32.8 & 15.6 & 44.5 & 36.4 & 4.39 & 1.9 \\
$\mathrm{CoZrNb}$ & 31.3 & 11.2 & 44.3 & 34.8 & 4.59 & 1.98 \\
$\mathrm{NiFeNb} / \mathrm{CoZrNb}$ & 31.8 & 12.1 & 44.5 & 34.9 & 4.93 & 1.85 \\
Exchange-pinned & 33.5 & 15.3 & 45.3 & 35.9 & 4.31 & 1.95 \\
$\mathrm{NiFeNb}$ & & & & & & \\
\hline \hline
\end{tabular}

(a) NiFeNb

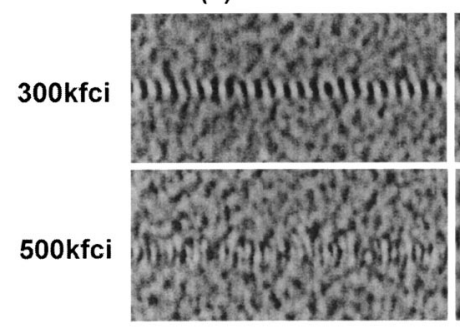

(b) CoZrNb

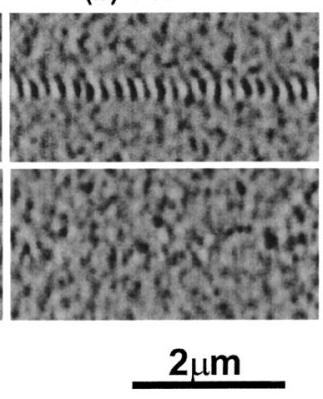

FIG. 2. MFM images of written tracks of 300 and $500 \mathrm{kfci}$ for media with (a) $\mathrm{NiFeNb}$ and (b) CoZrNb SULs.

media with $\mathrm{NiFeNb}$ and $\mathrm{CoZrNb}$ SUL, yet, $\mathrm{OW}_{6 \mathrm{~T} / 1 \mathrm{~T}}$ for media with $\mathrm{NiFeNb}$ is $1.6 \mathrm{~dB}$ higher than that with $\mathrm{CoZrNb}$ SULs, indicating the NiFeNb SUL has better writability than the CoZrNb SUL. Further experiments to confirm the writability effect on recording performance were done by sputtering a $50-\mathrm{nm}$-thick CoZrNb on top of a $200 \mathrm{~nm} \mathrm{NiFeNb}$. The media performance for this SUL combination was degraded compared to that with a single NiFeNb SUL. The writability of the SUL close to the recording layer is more important for achieving better recording performance. As for the exchange-pinned $\mathrm{NiFeNb}$, the recording performance is comparable to the NiFeNb SUL. This result cannot be simply explained by the permeability of the SUL since the permeability exchanged-pinned $\mathrm{NiFeNb}$ is greatly reduced compared to that of $\mathrm{NiFeNb}$.

Figure 2 shows the magnetic force microscope (MFM) images of written tracks of 300 and $500 \mathrm{kfci}$ for media with $\mathrm{NiFeNb}$ and CoZrNb SULs. As seen from the image, the $\mathrm{NiFeNb}$ SUL supports recording density patterns over 500 kfci and exhibits well-defined track edges in the $300 \mathrm{kfci}$ patterns. This is due to the improved head field gradient at high frequency writing from the higher permeable SUL.

Dynamic coercivity measurements were performed in the same way described in Ref. 5. Briefly, the medium was band dc erased prior to the experiment. Then, the test recording pattern was written using the optimized write head current value. The recorded track was then dc erased using low head erase current for one disk revolution. The residual track amplitude was then measured through a bandpass filter with a $100 \mathrm{kHz}$ bandwidth and the entire procedure was repeated using an incremented current starting from the complete dcband erasure of the test track. The half-current $I_{A / 2}$ is the current value required to reduce the track amplitude to $\frac{1}{2}$ of its initial as-recorded value and is proportional to the remanent coercivity $H_{\mathrm{cr}}$. The objective of the test was to find the half-current value for each incremented sweep time setting. Dynamic coercivity measurements at different recording frequencies showed that the recording frequency dependence of dynamic $H_{c}$ was also strongly affected by the writability of different SULs. Figure 3 shows $I_{A / 2}$ dependence on sweep time for typical longitudinal media and three PMR media with $\mathrm{CoZrNb}$, NiFeNb, and exchange-pinned $\mathrm{NiFeNb}$, respectively. In general, for PMR media, higher recording frequency patterns, which have lower demagnetizing fields, 

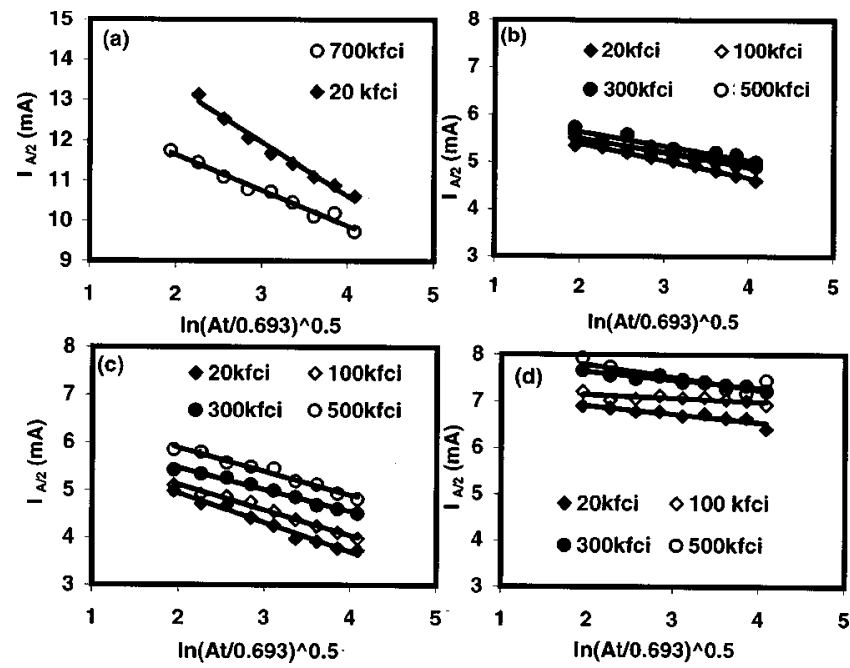

FIG. 3. Dynamic coercivity measurement on (a) longitudinal media; (b) PMR media with NiFeNb SUL; (c) PMR media with CoZrNb SUL; and (d) PMR media with exchange-pinned NiFeNb SUL.

show higher resistance to erasure. This is exactly opposite to the longitudinal media case where low recording frequency patterns, having lower demagnetization fields, show higher resistance to erasure [Fig. 3(a)]. As one can see, for media with a CoZrNb SUL [Fig. 3(b)], as the recording frequency increases, the time dependence of the coercivity sharply increases while the increase in dynamic $H_{c}$ with linear recording density is negligibly small for media with a NiFeNb SUL [Fig. 3(c)]. As for exchange-pinned NiFeNb, the increase in dynamic coercivity with increasing linear recording density becomes more pronounced due to the lower permeability. This indicates that an appropriate selection of the soft under- layer structure may result in a small difference between dynamic coercivity at different recording frequencies in perpendicular media. This is of extremely high importance for high density, high data rate applications. The above experiments also show that unlike longitudinal media whose writability is merely determined by recording layer, the SUL can change the writability for PMR media, adding versatility to media design.

\section{SUMMARY}

In summary, we have studied the recording characteristics of perpendicular media with different types of SULs. Our study shows that the recording performance depends on the permeability of SUL. Higher permeability NiFeNb shows better recording performance than the lower permeability $\mathrm{CoZrNb}$. Exchange-pinned $\mathrm{NiFeNb}$ suppresses spike noise and offers good recording performance. Dynamic coercivity measurements show that the writability offered by PMR media can be greatly improved by an appropriate selection of the soft underlayer composition and structure, giving PMR media another apparent advantage over longitudinal recording media.

\section{ACKNOWLEDGMENTS}

The authors would like to thank Dr. Ramamurthy Acharya for useful discussions and Philip Chung for the Guzik testing.

${ }^{1}$ H. S. Jung and W. D. Doyle, IEEE Trans. Magn. 37, 2294 (2001).

${ }^{2}$ T. Ando and T. Nishihara, IEEE Trans. Magn. 37, 1228 (2001).

${ }^{3}$ K. Tanahashi, A. Kikukawa, N. Shimizu, and Y. Hosoe, J. Appl. Phys. 91, 8049 (2002).

${ }^{4}$ J. Yu, C. H. Chang, D. Karns, G. P. Ju, Y. Kubota, W. Eppler, C. Brucker, and D. Weller, J. Appl. Phys. 91, 8357 (2002).

${ }^{5}$ M. Alex and D. Wachenschwanz, IEEE Trans. Magn. 35, 2796 (1999). 